\title{
Functional Similarities in Spatial Representations Between Real and Virtual Environments
}

\author{
BETSY WILLIAMS, GAYATHRI NARASIMHAM, CLAIRE WESTERMAN, JOHN RIESER, \\ and BOBBY BODENHEIMER \\ Vanderbilt University
}

\begin{abstract}
The two experiments in this paper demonstrate similarities in what people know about the spatial layout of objects in familiar places whether their knowledge resulted from exploring the physical environment on foot or exploring a virtual rendering of it with a tethered head-mounted display. In both experiments subjects were asked to study the locations of eight targets in the physical or virtual environment, then close their eyes, walk (or imagine walking) to a new point of observation, and then turn and face some of the remembered objects. In Experiment 1 the results of the statistical analysis were functionally similar after learning by exploring the virtual environment and physical environment: The new points of observation were simple rotations of the point where they were learned. Like exploring the physical environment, the latencies and errors of the turning judgments after learning the virtual environment were significantly worse after the imagined movements than the physical movements; and the measures were worse for larger degrees of physical or imagined rotation in both conditions. In Experiment 2 the new points of observation differed by simple rotations in one condition versus simple translations in the other condition and again the results were functionally similar after learning the physical versus the virtual environment: In both learning conditions, the errors and latencies in the physical and virtual environments were worse after rotations than translation and varied as a function of the disparity of the direction when learning and the direction when responding.
\end{abstract}

Categories and Subject Descriptors: I.3.7 [Computer Graphics]: Three-Dimensional Graphics and RealismVirtual Reality

General Terms: Experimentation, Human Factors, Measurement

Additional Key Words and Phrases: Virtual reality (VR), space perception

\section{INTRODUCTION}

An important application of virtual environments is based on the assumption that what people learn from exploring physical environments is functionally similar to what they learn from exploring virtual renderings of them. Such functional similarity is important when-

Authors addresses: Williams, Bodenheimer: Electrical Engineering and Computer Science, Vanderbilt University, VU Station B \#351679, 2301 Vanderbilt Place, Nashville, TN 37235-1679; email: \{betsy.williams,bobby.bodenheimer\}@vanderbilt.edu. Narasimhan, Westerman, Rieser: Department of Psychology and Human Development, Peabody \#512, 230 Appleton Place, Nashville, TN 37203-5721; email: \{gayathri.narasimham,claire.b.westerman,john.j.rieser.2\}@ vanderbilt.edu.

This research was supported by National Science Foundation Grants IIS-0237621 and IIS-0121084.

Permission to make digital/hard copy of all or part of this material without fee for personal or classroom use provided that the copies are not made or distributed for profit or commercial advantage, the ACM copyright/server notice, the title of the publication, and its date appear, and notice is given that copying is by permission of the ACM, Inc. To copy otherwise, to republish, to post on servers, or to redistribute to lists requires prior specific permission and/or a fee.

(c) 2006 ACM 0700-0234/2006/0100-0001 $\$ 5.00$ 
ever people hope to explore and learn about virtual environments to apply their knowledge when planning or acting in the physical environments that they represent. Examples abound where evaluating scenarios in the physical world would be expensive and difficult so that virtual environments are used instead - for example — in simulations of behindthe-lines military operations, piloting a ship or plane, or learning to navigate a human body to prepare for surgery. The aim of the two experiments presented here is to assess the similarities and differences in how and how accurately people judge the directions toward objects after learning their locations by exploring physical environments versus virtual renderings of them.

To illustrate these similarities, note that after exploring and learning about physical environments, people can judge the perspectives available at various locations within the familiar place, even if the perspective is one that they have never directly experienced [Rieser et al. 1994]. Consider three conditions that influence the relative ease of such judgments. First, judging new perspectives is done more effectively when people physically locomote as if moving to the new perspective. This situation occurs when people close their eyes and locomote relative to their actual remembered surroundings [Loomis et al. 1999; Rieser et al. 1986b]. In addition, when people stand in one place and pretend to be somewhere else, they are able to imagine new perspectives [Rieser et al. 1994; May 1996; 2004]. Second, people find it easier to judge changes in perspective when the geometry of the change is a translation rather than a rotation [Rieser 1989; Presson and Montello 1994; Philbeck et al. 2001]. Third, the difficulty of making a perspective change is a monotonic function of the degree of disparity between the original perspective and the to-be-judged perspective [Rieser 1989; May 2004]. By disparity we mean the difference in direction between targets from the new, to-be-judged point of observation and the one from which they were originally learned, a measure discussed further in Section 2.

Two experiments are reported here in which subjects were asked to judge new perspectives across variations in locomotion mode (physical or imagined), geometry of perspective change (translation or rotation), and amount of disparity. In both experiments, subjects learned a novel environment by freely exploring it in each of two visual input conditions: in one people viewed their actual physical surroundings and in the other they viewed a graphically rendered simulation of the same surroundings presented over a head-mounted display (HMD). The purpose of the experiments was to investigate the functional similarity of the representations derived from viewing the physical environment and the virtual environment.

By functional similarity, we mean similarities or differences in performance when judging new perspectives based on the locomotion mode, the geometry of the perspective change, and the amount of disparity. These judgments were assessed by measuring the errors and latencies (time to complete the task) of the perspective changes. Both measures are free to vary in our experiments and both provide a measure of subjects' access to their spatial knowledge gained through one of the visual input modes.

The immersive qualities of virtual environments are getting better and ultimately we expect there to be no significant differences in operating on knowledge gained by exploring and learning about physical environments and their virtual renderings (immersion is a concept difficult to define, but see [Witmer and Sadowski 1998]). However, current HMD technology (the limits of our particular HMD are discussed in Section 4.1.2) is limited in expressing rich visual representations. For example, several groups have reported inaccura- 
cies of judging distances in immersive environments [Loomis and Knapp 2003; Thompson et al. 2004; Willemsen and Gooch 2002; Witmer and Sadowski 1998]. A number of factors may contribute to this discrepancy, such as the limited field of view, spatial resolution, subtle errors in rendering, and the weight of the HMD.

Thus, we expect performance judging perspectives after exploring the virtual environment over the HMD to be less accurate or slower to process than after viewing the physical environment. Hence, a significant or not significant effect of the visual input type on these experiments is interesting, and potentially indicates the quality of immersion, but is not the primary goal of our work. Instead, an analysis of functional similarities involves determining whether the conditions that influence responses from representations learned while viewing the physical environment have similar influence on responses from representations learned while viewing virtual environments.

This paper is organized as follows. Section 2 discusses related work and places the present work in context. The research in this paper consisted of two main experiments, and the scope and design of these experiments is explained in Section 3. The first experiment is described and its results reported in Section 4; the second is described and results reported in Section 5. We assess the impact of these experiments in Section 6.

\section{BACKGROUND}

Studies on human behavior [Loomis et al. 1999; Plumert et al. 2004] can be conducted in virtual environments. This paper examines imagined and physical rotation in real and virtual environments. Many studies [Easton and Sholl 1995; Farrell and Robertson 1998; May 1996; Presson and Montello 1994; Rieser 1989; Rieser et al. 1986a; Wraga 2003] have shown that updating spatial orientation is much harder with imagined movement than physical locomotion. In [Rieser 1989], subjects were asked to point to a target after imagining facing an object and after physical locomotion to an object. The study showed that performance was slower for imagined rotations than physical rotations, and the response latency for the imagination condition increased as a function of the angle from the participant's facing direction to the imagined location. A possible explanation is that imagined rotations lack proprioceptive feedback. We intend to measure similar responses for the virtual environment and compare them to the physical environment.

In this study, we also compare two different imagined geometric movements, translation, i.e., changes in self location keeping the current facing direction, and rotation, i.e., changes in current facing direction while retaining the same location. Subjects are tested in both the physical and virtual worlds to test for functional similarity. A number of studies, [Easton and Sholl 1995; May 2004; Presson and Montello 1994; Rieser 1989], have shown imagined translations are faster and more accurate than imagined rotations. Furthermore, many have shown that response times and errors increase as a function of the angle of imagined rotation [Easton and Sholl 1995; Rieser 1989; Wraga 2003]. In these experiments, translations and rotations are decoupled, in contrast to other work, e.g., [Klatzky et al. 1998].

This paper uses a spatial orientation task where subjects turn to face a direction, similar to the pointing task of [Rieser 1989]. Other comparisons of spatial updating and pointing in virtual environments have been done, although none have replicated the conditions of the present work. [Chance et al. 1998] report that for subjects walking through a virtual maze, physical translation and rotation allowed subjects to update better than physical translation 
and joystick rotation. [Klatzky et al. 1998] report that optic flow without locomotion in an HMD was not sufficient to induce spatial updating for turn responses, although [Riecke et al. 2005] report the opposite result for large field-of-views. [May and Klatzky 2000] find a functional similarity between real and virtual environments in that in both environments irrelevant movement had greater effects on a path integration task than verbal or cognitive distractions. [Wraga et al. 2004] also studied spatial updating in virtual displays and report that active rotation, in which the subjects rotate themselves, has advantages over passive rotation, in which the subjects were rotated by the experimenter. We note that they thus break down locomotion in two ways that we do not. In our first experiment, subjects were passively rotated, while in our second, subjects actively rotated. However, the advantage found by [Wraga et al. 2004] was small and we did not pursue that classification of rotation further. [Waller et al. 2004] examined directional knowledge in the real world and virtual environments and also found a functional similarity between real and immersive HMD environments. Thus, the prevailing view in the literature seems to be that there are similarities between physical and virtual environments, and that locomotion in a virtual environment can help spatial updating, two views that we explore further in this paper.

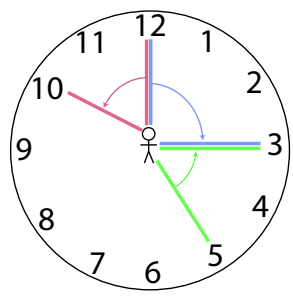

Fig. 1. An example of rotational disparity. The subject is facing the 12 . The instruction "turn to face the 3 as if facing the 5 " is given. To give the correct turn response to this example, the subject must turn to the left $60^{\circ}$ as shown in red. The disparity is the difference between the real facing direction (12) and the target (3), $90^{\circ}$ (blue), and the imagined facing direction (5) and target (3), $-60^{\circ}$ (green). Thus, the disparity is $90-(-60)=150^{\circ}$. The disparity is also equal to the difference between the final and facing position (10) and the target (3).

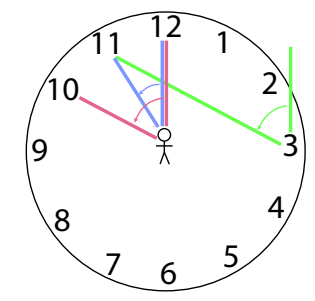

Fig. 2. An example of translational disparity. The subject is facing the 12 when the instruction "turn and face the 11 as if standing at the 3 " is given. The correct motion is to turn to face the 10 (shown in red). The disparity is the difference between the real facing direction (12) and the target (11), $-30^{\circ}$ (shown in blue), and the imagined facing direction (3) and target (11), $-60^{\circ}$ (shown in green). Thus the disparity is $-30-(-60)=30^{\circ}$. This quantity is also the same as the difference between the new facing direction (10) and the target (11).

[May 2004] argues that response latencies and errors due to perspective change are related to the conflict between the two perspectives. Similar research, [Brockmole and Wang 2003; May 1996; Newcombe and Huttenlocher 2000; Wang 2005] has supported this interference hypothesis. This effect is a function of a quantity called disparity, which is illustrated in Figures 1 and 2. Disparity is defined as the difference in angle when turning to face a given target relative to one's actual position in the physical or virtual room from the angle to-be-turned given the to-be-imagined facing direction (the location where the object is thought to be). In Figure 1, the subject is facing the 12, and then imagines rotating to the 5. Now the subject is asked to point to the 3 . When the subject is facing the 12 , 
the 3 is on his right; when the subject is facing the 5 , the 3 is on his left. These two relationships will interfere with each other as the subject makes a decision about the correct location of the 3 facing the 5 . The effects of this interference increase as the magnitude of this difference, the disparity, increases. In this paper, we use the term "response disparity" or simply "disparity" to mean the "object direction disparity" as defined by [May 2004]. For rotations, the amount of disparity is equal to the angle of imagined rotation as shown in Figure 1. For translations, the amount of disparity is equal to the difference between the angle from the facing direction to target object, and the angle from the imagined source object location to target object as shown in Figure 2.

Our work discusses aspects of the relation between perception, representation, and action. Some investigation of the relation between perception and action in virtual environments has been conducted, e.g., [Mohler et al. 2004; Mohler et al. 2005; Kay and Warren, Jr. 2001]. In particular, much recent work [Loomis and Knapp 2003; Thompson et al. 2004; Willemsen and Gooch 2002; Witmer and Sadowski 1998], has studied the issue of the similarities and difference in distance estimation between real and virtual environments. This work has found that subjects underestimate distances in virtual environments. The precise reasons for this are not known, but several factors have been examined. There have been different empirical findings on how field of view in an HMD leads to an underestimation of distance. [Wu et al. 2004] show that vertical field of view, FOV, of $21^{\circ}$ or less leads to an underestimation of distance. [Knapp and Loomis 2004] found that a reduced vertical FOV similar to that of an HMD has no influence in the real environment. The weight of the HMD itself may also cause problems with distance perception [Willemsen et al. 2004]. [Thompson et al. 2004] show that distance perception in real and virtual environments is not due to the lack of realistic graphics. While these distance discrepancies exist in the virtual environment seen though the HMD, [Plumert et al. 2005] found that time to walk estimates in real environment and virtual large-screen immersive display environment were highly similar. [Oman et al. 2002] tested the ability of subjects to learn objects' spatial relationship and to predict their location as their bodies were specified in different 3D orientations. They found that body positions with respect to gravity had a minor but significant effect on locating objects, and that performances in the real world were functionally equivalent to those in the virtual. In contrast to the above, our work looks at the relation between perception and representation.

\section{DESIGN OF THE STUDIES}

Two experiments were designed to help evaluate the functional similarities in using knowledge learned from exploring a large room-sized physical environment and a virtual construction of the same environment presented over an HMD and explored freely on foot. In each experiment subjects were asked to explore and learn the spatial layout of eight target objects. After learning the target locations from the perspective at one point of observation, they were asked to close their eyes and make knowledge-based judgments of the target directions from a new perspective. An important note about these experiments is that we did not restrict the FOV of a subject in the physical environment to be the same as in the virtual environment, as some have done, e.g., [Thompson et al. 2004]. The reason for our decision is that we wanted to test our subjects when they learned in settings that were as unencumbered as possible. For a virtual environment presented through an HMD, this setting involves limitations on FOV, but does not in the physical environment. Thus, the 
emphasis in these studies is on the functional similarities in spatial reasoning despite the many differences in input for learning.

Experiment 1 was designed to determine whether physical locomotion facilitates the speed and/or accuracy of judging new perspectives after simple rotations in facing direction. In the locomotion condition the subjects were blindfolded (or the HMD was darkened), they were physically guided to face the new direction, and they were asked to judge the target directions. In the imagination condition the subjects were blindfolded and asked to imagine turning to face the new direction. Thus, the three factors of the study were visual input (visually exploring the physical environment versus its virtual environment rendition) $\times$ locomotion method (physical locomotion versus imagination) $\times$ disparity (that is, there were eight different amounts of rotation in perspective to be judged, ranging from $0^{\circ}$ to $180^{\circ}$ ). The type of visual input was varied across subjects (one-half studied the physical environment and one-half studied the virtual environment), the locomotion method was blocked and varied within repeated trials of each subject, and the disparity was randomly ordered within each block for each subject. The blocking adds an additional condition that allows us to assess whether learning occurs between blocks.

Experiment 2 was designed to determine whether the geometry of the imagined changes in perspective (there was no physical locomotion) influence the difficulty. Again subjects visually explored either a physical room or a virtual rendering of the same room and then were asked judge target directions after imagining changes in perspective that consisted of simple rotations (that is, subjects were asked to imagine they had turned to change their facing direction while standing in the same spot in the room) or simple translations (that is, subjects were asked to imagine keeping their facing direction constant while moving straight to a new spot in the room). The amount of to-be-imagined change in facing direction varied across both the rotation trials and the translation trials. The type of visual input was varied across the subjects, the geometry of the perspective change was blocked within the repeated trials of each subjects, and the disparity was randomly varied within these blocks. The blocking was again a condition within the experiments that we consider.

People typically move through their environment using a combination of both rotation and translation, and Experiment 2 decouples them. In comparing translations and rotations, the underlying difficulty of the a perspective change depends on the disparity, as discussed previously. In the type of experiment we designed (modeled after the studies of [Rieser 1989]), disparities for both translation and rotations fall into natural and principled values. However, these values are not identical, and, thus for our design, the range of disparities for translations will be less than the range of disparities for rotations, and the natural values of disparities for translations will be different than the natural values of disparities for rotations. When comparing rotational and translational disparities prior work (e.g., [May 2004]) has typically clustered or "binned" values. Statistical power can be lost through this binning, and modern statistical methods [Harrell, Jr. 2001; Myers et al. 2002] do not require binning. Thus, our analyses in later sections will use the true, unbinned values of disparity. However, for didactic purposes, we display binned values of disparity in the figures, as it is easier to visually interpret this style of presentation than a scatterplot, and, as we will see, doing so does not misrepresent the data.

Thus, both experiments were designed to find out the relative difficulty of knowledgebased judgments of perspective changes as a function of whether the knowledge was learned from visually exploring a virtual environment versus a physical one. In addition, 
they allow us to investigate the degree to which the relative difficulty of the judgments was influenced by whether the perspective change was accompanied by locomotor movements, by the disparity in perspective that was to be judged, and by whether the geometry of the change consisted of a simple rotation or simple translation in perspective. To assess relative difficulty, we asked subjects to turn and face the target objects from the new perspectives quickly and accurately. Both the turning errors and the latencies can be expected to vary as a function of difficulty, except in situations where subjects trade speed for accuracy by, for example, responding rapidly and inaccurately some of the time and slowly and accurately some of the time. As long as latencies and accuracies are positively correlated (that is, people are not trading speed for accuracy), then both are reasonable measures of relative difficulty. However, in conditions where latencies and accuracies are negatively correlated, then the meaning of both measures is ambiguous and cannot be used to sort the relative difficulty across conditions.

\section{EXPERIMENT 1}

In this experiment, our goal was to assess similarities of spatial knowledge between physical and virtual environments. More specifically, for both environments, we investigate the relative difficulty of imagining rotations and whether physical rotation facilitates access to spatial structure. This experiment mimics Experiment 2 of [Rieser 1989] with the additional condition of being conducted in a virtual or physical environment.

\subsection{Method}

4.1.1 Participants. Sixteen Vanderbilt University students participated in the experiment. Subjects were unfamiliar with the experimentation room and the virtual reality equipment.

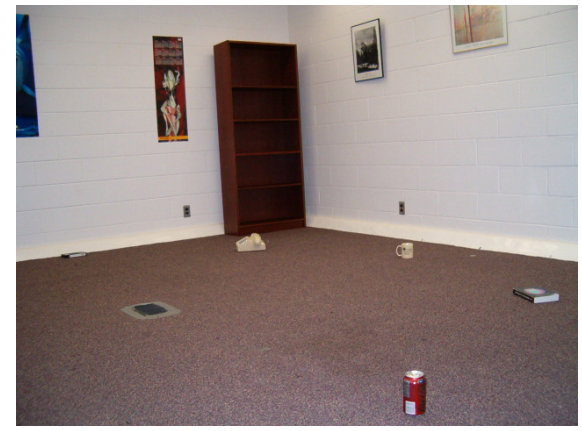

Fig. 3. Laboratory or "physical" environment for conducting experiments. Shown is the circular array of eight objects used in the experiments. For both Experiment 1 and Experiment 2, subjects stand in the center of the array. This particular environment was used in Experiment 2, and Experiment 1's virtual environment was similar.

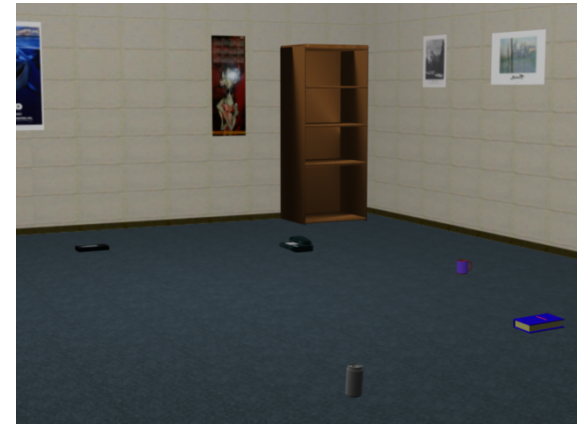

Fig. 4. This figure shows the virtual environment and the circular array of eight objects used in the experiments. 
4.1.2 Materials. In both the real and virtual conditions, participants viewed eight targets (ball, rubber duck, bottle, telephone, vase, videotape, scissors, and clock) that were arranged in an evenly spaced circle. Each of the targets varied in shape and color. Identical objects in the same location were used for the virtual and real world condition, and were of similar size. A virtual room, as seen in Figure 4, of the same scale and layout was designed to mimic the real environment, shown in Figure 3 (the views are not exactly similar because we could not replicate the exact camera parameters between the physical and virtual cameras).

The virtual world was viewed through a full color stereo Virtual Research Systems V8 HMD with $640 \times 480$ resolution per eye, a field of view of $60^{\circ}$ diagonally, and full binocular overlap. The HMD also had an angular resolution of approximately $2.2^{\circ}$, and weighed approximately $1 \mathrm{~kg}$. An InterSense IS-900 tracker was used to update the participant's rotational movements. The rendered field of view was matched to the displayed field of view (otherwise, performance errors can result [Psotka et al. 1998]).

4.1.3 Procedure. One-half of the subjects performed the experiment in the virtual world, and the other half performed the experiment in the real world. In both conditions, subjects stood in the center of the array of objects, which was roughly the center of a $5.8 \mathrm{~m}$ by $6.4 \mathrm{~m}$ room. Each target was approximately $2 \mathrm{~m}$ from the participant. After approximately three minutes of study, the experimenter tested the subjects by asking them to close their eyes and point to randomly selected target objects. This testing and learning procedure was repeated until both the experimenter and subject were confident that the configuration had been learned.

Next participants were asked to imagine themselves at a novel direction of observation, and point to a target from that point. Specifically participants were instructed "face the \langle target name $\rangle$ as if you are facing the $\langle$ source object name $\rangle$." There were two conditions: a locomotion condition and an imagination condition. In the locomotion condition, subjects were physically turned by the experimenter to face the source object during the verbal instruction. To move the subject, the experimenter grasped the subject by the shoulders and rotated him. In the imagination condition, subjects followed the instruction by imagining turning to face the object that identified the new facing direction. Note that in some of the trials, the subject was actually facing the source object, so that no imagined or physical locomotion occurred. Subjects were asked to not simply turn their heads, but to locomote their entire body, although the response was measured from the HMD based on the headfacing direction. Note also that as we measured response angles through the HMD, in all conditions subjects wore a darkened HMD when turning, which served as a blindfold. The instructions for the imagination and locomotion conditions were explained before participants saw the experimental layout. After every trial, subjects rotated themselves back to a starting position facing the front of the room. If there was any error after this rotation between their facing direction and the correct starting position the experimenter rotated them to the correct starting position. The subjects completed 56 test trials for each condition in two blocks of 28. The order of the instructions for each block was randomized. Half of the subjects were tested first for imagination, and the other half were tested first for the locomotion condition. To compare the angles of the correct responses across different conditions, the same trials were used for the locomotion and imagination modes, and for the virtual and real world environments.

The subjects indicated to the experimenter that they were facing the target by verbal 
instruction, and the experimenter recorded their time and rotational position. The time was recorded using a stopwatch, and the rotational position was recorded using the InterSense tracker. Subjects were encouraged to respond as rapidly as possible, while maintaining accuracy. If subjects were confused, they were allowed to look again at the array during the experiment, between trials.

\subsection{Results}

The dependent variables in this experiment were the errors and latencies in turning to face the targets. There were four independent variables: visual input type, locomotion method, amount of disparity, and the learning block (either first or second) in which the particular trial was conducted. The type of input was either virtual or physical, depending on whether the subject learned the target locations in the virtual space or physical space. The locomotion method was either imaginary (imagined rotation) or physical (physically turned). The amount of rotation varied as repeated within-subject trials. We analyze results by learning block to determine if order effects (i.e., learning) are an important factor in the experiment.

Figure 5 shows the mean turning error, and Figure 6 shows the mean latencies as functions of visual input mode and locomotion method. Turning error was defined as the unsigned error, i.e., the unsigned difference between the actual target distances and the observed distances. The average turning errors as functions of disparity and locomotion method are presented separately for the physical input mode, Figure 7, and the virtual input mode, Figure 8. Likewise, the average latencies as a function of disparity and locomotion method are shown in Figure 9 (physical input mode), and Figure 10 (virtual input mode). Note that disparity is a continuous variable and has values between 0 and 180 determined by the geometry of the experimental setup. Following the practice of [May 2004], for ease of presentation in these figures we have clustered the disparities to their closest $45^{\circ}$ amount. In the statistical analysis described below, however, we use the unclustered "true" disparity values. The mean turning error and latency by learning block are shown in Figures 11 and 12 , respectively.

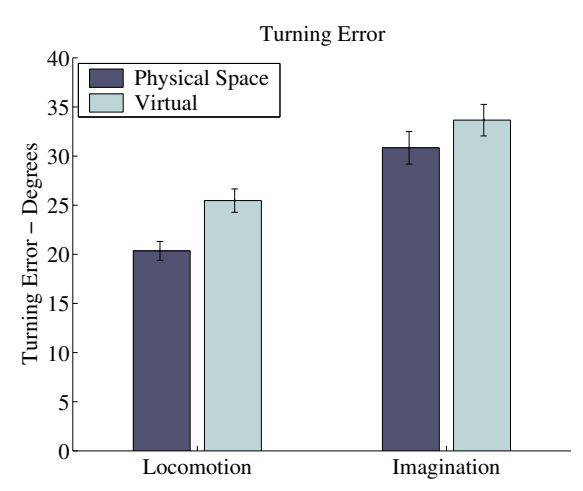

Fig. 5. Mean turning error for both the locomotion and imagination method in the virtual and physical conditions in Experiment 1. Error bars indicate the standard error of the mean.

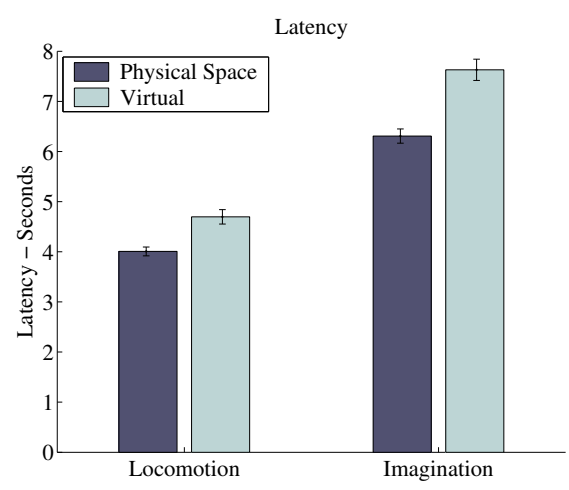

Fig. 6. Mean latency for both the locomotion and imagination task in the virtual and physical conditions in Experiment 1. Error bars indicate the standard error of the mean. 


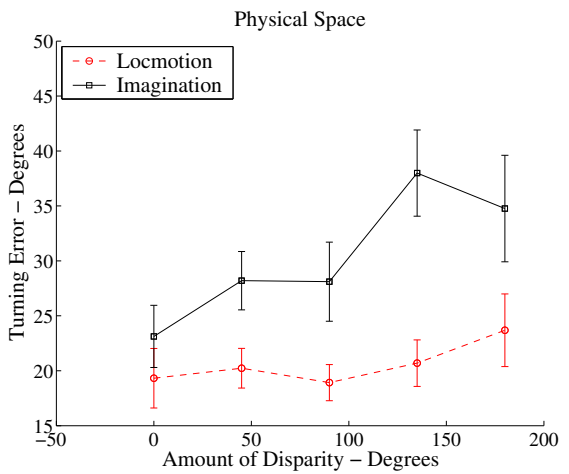

Fig. 7. Mean turning error as a function of disparity in the real world condition for Experiment 1. The red line represents the turning error in the locomotion repositioning task; the black line shows the turning error in the imagination repositioning task. Error bars indicate the standard error of the mean.

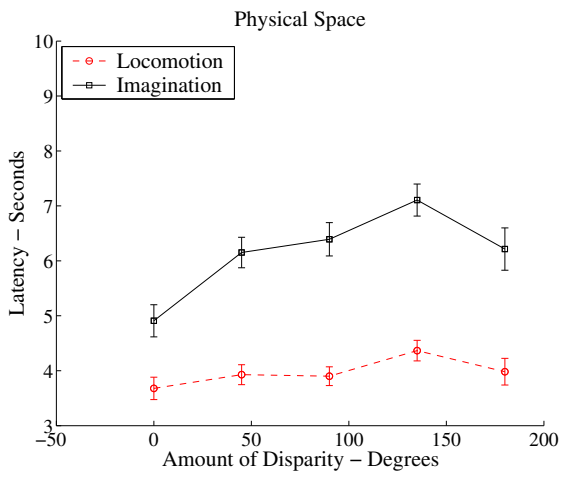

Fig. 9. Latency as a function of disparity in the real world condition for Experiment 1. The red line represents the latency in the locomotion repositioning task; the black line shows the latency in the imagination repositioning task. Error bars indicate the standard error of the mean.

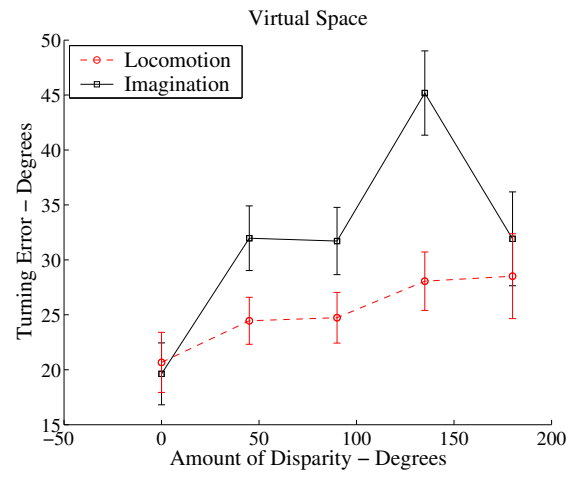

Fig. 8. Mean turning error as a function of disparity in the virtual condition for Experiment 1. The red line represents the turning error in the locomotion repositioning task; the black line shows the turning error in the imagination repositioning task. Error bars indicate the standard error of the mean.

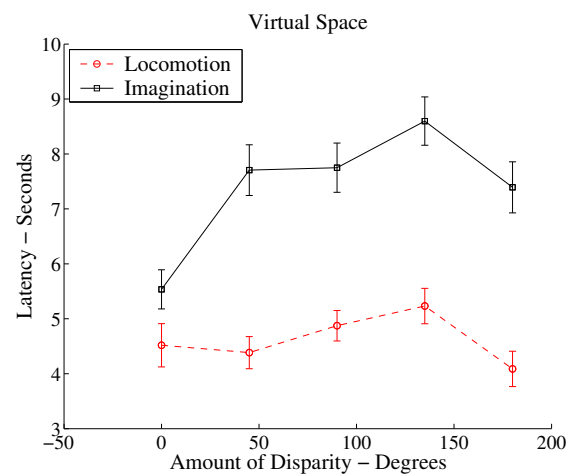

Fig. 10. Latency as a function of disparity in the virtual condition for Experiment 1. The red line represents the latency in the locomotion repositioning task; the black line shows the latency in the imagination repositioning task. Error bars indicate the standard error of the mean.

Since the amount of disparity is a quantitative independent variable, we used least squares regression to fit a generalized linear (mixed) model to the data, and conducted an analysis of variance to test the significance of the independent variables and associated interactions using techniques described in [Harrell, Jr. 2001; Myers et al. 2002]. We used the four independent variables to predict the turning error and latency, respectively, and modeled all two-way and three-way interactions of the independent variables. The resulting model had 14 degrees of freedom. This type of model allows us to test if disparity was better modeled as a quadratic, but the quadratic effect was not significant. Tables I and II show the analysis of variance results for this experiment. Results were considered 

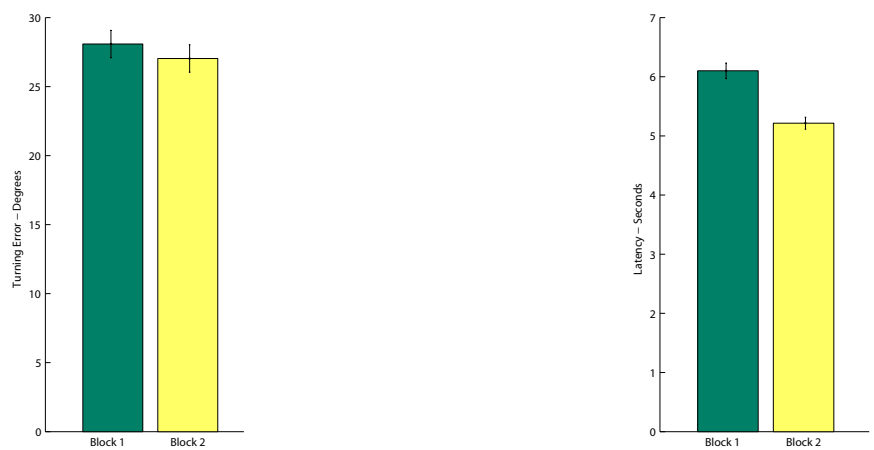

Fig. 11. This figure shows the mean turning error in the first block and second block of Experiment 1. The error bars represent standard errors of the mean.
Fig. 12. This figure shows the mean latency in the first block and second block of Experiment 1. The error bars represent standard errors of the mean.

\begin{tabular}{lrrrrr}
\hline \hline & $d . f$. & PartialSS & \multicolumn{1}{c}{$M S$} & \multicolumn{1}{c}{$F$} \\
\hline Disparity* & 7 & 26732 & 3819 & 4.5 & $<.01$ \\
Visual Input & 7 & 11319 & 1617 & 1.9 & .06 \\
Locomotion* & 7 & 53921 & 7703 & 9.1 & $<.01$ \\
Block* & 7 & 12789 & 1827 & 2.2 & .03 \\
Disparity $\times$ Visual Input & 3 & 2965 & 988 & 1.2 & .32 \\
Disparity $\times$ Locomotion & 3 & 5780 & 1927 & 2.3 & .08 \\
Visual Input $\times$ Locomotion & 3 & 764 & 255 & 0.3 & .82 \\
Disparity $\times$ Block & 3 & 2196 & 732 & 0.9 & .46 \\
Visual Input $\times$ Block & 3 & 2710 & 903 & 1.1 & .36 \\
Locomotion $\times$ Block* & 3 & 9414 & 3138 & 3.7 & .01 \\
Disparity $\times$ Visual Input $\times$ Locomotion & 1 & 2 & 2 & 0.0 & .97 \\
Disparity $\times$ Visual Input $\times$ Block & 1 & 1928 & 1928 & 2.3 & .13 \\
Disparity $\times$ Locomotion $\times$ Block & 1 & 11 & 11 & 0.0 & .91 \\
Visual Input $\times$ Locomotion $\times$ Block & 1 & 289 & 289 & 0.3 & .56 \\
\hline
\end{tabular}

Table I. Analysis of Variance for Turning Error in Experiment 1. The $d . f$. column gives the number of coefficients of terms including that term in the regression model. Other columns are standard statistical measures. Significant main effects and interactions are indicated with an asterisk $(*)$.

significant if $p<.05$. The "effective" degrees of freedom are reported [Satterthwaite 1941; 1946]. Since correlation may exist over repeated values of disparity, the residual errors in the regression may not be independent, and thus the variance estimates may be wrong. To correct this problem, the degrees of freedom are adjusted.

From these tables, we see that there are a significant main effects of disparity, locomotion mode (physical or imagined), and learning block on the turning errors and latencies. The main effect on disparity indicates that lower disparities lead to more accurate, faster performance. Subjects were faster and more accurate in their judgments when turned (the locomotion mode) than when they have to imagine turning. Also, the performance of subjects in block 2 was faster and more accurate than performance in the block 1.

For turning error, there is a significant interaction of locomotion on learning block; turning errors decreased in the physical locomotion condition from block 1 to block 2 , while they increased slightly in the imagination condition from block 1 to block 2 . Subjects 


\begin{tabular}{lrrrrc}
\hline \hline & $d . f$. & PartialSS & $M S$ & $F$ & $p$ \\
\hline Disparity* & 7 & 329 & 47 & 4.6 & $<.01$ \\
Visual Input* & 7 & 557 & 80 & 7.9 & $<.01$ \\
Locomotion* & 7 & 3243 & 463 & 45.8 & $<.01$ \\
Block* & 7 & 488 & 70 & 6.9 & $<.01$ \\
Disparity $\times$ Visual Input & 3 & 8 & 3 & 0.3 & .85 \\
Disparity $\times$ Locomotion* & 3 & 103 & 34 & 3.4 & .02 \\
Visual Input $\times$ Locomotion & 3 & 56 & 19 & 1.9 & .13 \\
Disparity $\times$ Block & 3 & 10 & 3 & 0.3 & .81 \\
Visual Input $\times$ Block & 3 & 57 & 19 & 1.9 & .13 \\
Locomotion $\times$ Block & 3 & 64 & 21 & 2.1 & .10 \\
Disparity $\times$ Visual Input $\times$ Locomotion & 1 & 5 & 5 & 0.5 & .49 \\
Disparity $\times$ Visual Input $\times$ Block & 1 & 3 & 3 & 0.3 & .57 \\
Disparity $\times$ Locomotion $\times$ Block & 1 & 0 & 0 & 0.0 & .87 \\
Visual Input $\times$ Locomotion $\times$ Block & 1 & 9 & 9 & 0.9 & .35 \\
\hline
\end{tabular}

Table II. Analysis of Variance for Latency in Experiment 1. The $d . f$. column gives the number of coefficients of terms including that term in the regression model. Other columns are standard statistical measures. Significant main effects and interactions are indicated with an asterisk $(*)$.

seemed to benefit from the chance to learn in the locomotion condition (performance improved), but in the more difficult imagination condition, subjects seemed to become fatigued and their errors increased. This interaction therefore does not seem to add much to our interpretation of the main effect.

For the latencies there is a significant main effect of visual input (real world or virtual). This effect is only marginal for the turning errors. Subjects were slower in completing the task if their visual input was through the HMD rather than through the physical environment. We discuss this further in the conclusions, but this effect may be due to various factors involving the immersion that the subjects felt in the virtual environment. There is a significant interaction between disparity and locomotion, meaning that disparity has a larger impact in the imagination condition than in the physical locomotion condition. This interaction is one we expect and consistent with the literature, e.g., [May 2004]. In particular, subjects had more difficulty turning accurately and speedily in the imagination condition than the locomotion condition for both environments, and the amount of difficulty they had depended on the amount of disparity in the task. The main conclusion we draw from this experiment is that there exists a functional similarity in subject's access to spatial knowledge in both real and virtual environments.

\section{EXPERIMENT 2}

In this experiment, we replicated and elaborated the design of Experiment 3 from [Rieser 1989]. Subjects were asked to learn the spatial layout of targets either by exploring a physical environment or a virtual rendering of it via a tethered HMD. Like the earlier experiment, subjects were asked to judge self-to-object directions from novel points of observation that were either simple rotations of their original point of simple translations of it.

\subsection{Method}

5.1.1 Participants. Fourteen Vanderbilt University students and two non-student adults participated in the experiment. All were unfamiliar with the experimentation room and the 
virtual reality equipment.

5.1.2 Materials. The HMD and tracker used in Experiment 1 were used again in Experiment 2. The array of objects used in this experiment were a sneaker, a videotape, a book, a hairbrush, a vase, a telephone, a soda can, and a coffee mug. These objects existed in the same locations in both the real and virtual environments as seen in Figures 3 and 4, and were spaced in 45 degree increments in a circle about $7 \mathrm{ft}$ from the center. Again, the room was approximately $17 \mathrm{ft}$ by $19 \mathrm{ft}$.

5.1.3 Procedure. One-half of the subjects performed the task in the virtual world, the other half in the physical world. In both the virtual and physical environments, there were two conditions: rotation and translation. Subjects imagine translating or rotating to a specific spatial point, then they are asked to face an object from that spatial location. The rotation condition was the same as Experiment 1, and participants were asked to "face the $\langle$ target name $\rangle$ as if you are facing the $\langle$ source object name $\rangle$. " In the translation condition, subjects were asked to "face the $\langle$ target name $\rangle$ as if standing at the $\langle$ source object name $\rangle. "$ The participants were tested using four alternating blocks of 28 trials. One-half of the subjects did a rotation block first, the other half did a translation block first. The procedures of the study were carefully explained to the subjects before they entered into the test room. Once the experimenter thought that the subject demonstrated understanding of the tasks, the subjects were led into the center of the array with their eyes closed. Next, the subjects were instructed to open their eyes and learn the locations of the objects in the array. The study phase was similar to that of the previous experiment and lasted about 2-5 minutes until the experimenter felt that the subject was familiar with the locations of the objects.

\subsection{Results}

Again in this experiment, the dependent variables were the errors and latencies in turning to face the targets, defined as in Experiment 1. There were four independent variables: visual input type, geometry of perspective change, amount of disparity, and the block (either first or second) in which the particular trial was conducted. Visual input, disparity, and block were as in Experiment 1 . The geometry of perspective change indicates whether the subject was asked to conduct a perspective change by turning (rotation) or by linear movement while maintaining heading direction (translation). Both the amount of rotation and the direction of translation varied as repeated within-subject trials. We again analyze results by learning block to determine if order effects (i.e., learning) are an important factor in the experiment. Data from two of the subjects were not calculated in the results as they failed to understand the task after repeated explanation. This failure was evident in comments they made after the experiment, and was evident in that they both performed worse than chance in the rotation and translation conditions.

Figures 13 and 14 show the mean turning errors and latencies, respectively. Mean turning error as a function of disparity is shown in Figures 15 and 16. Mean response time as a function of disparity is shown in Figure 17 and 18. In the figures, we again cluster the disparities into groups for purpose of presentation, but analysis results are done on the measured values. Note that the circular arrangement of objects constrains the maximum translational disparity that can occur, in contrast to arrangements in [May 2004]. Finally, mean turning error and latency as a function of learning block are shown in Figures 19 and 20. 


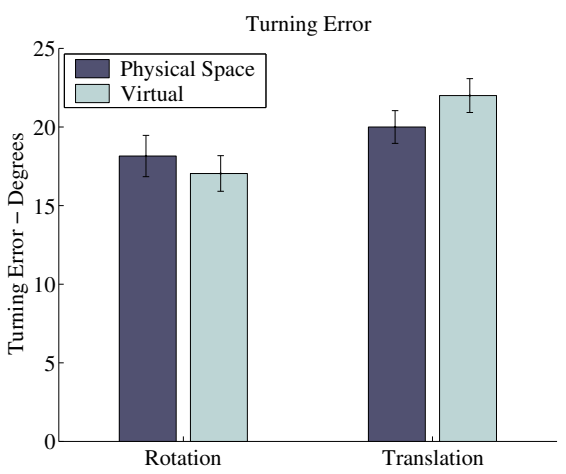

Fig. 13. Mean turning error for both the translation and rotation repositioning task in the virtual and real conditions for Experiment 2. Error bars indicate the standard error of the mean.

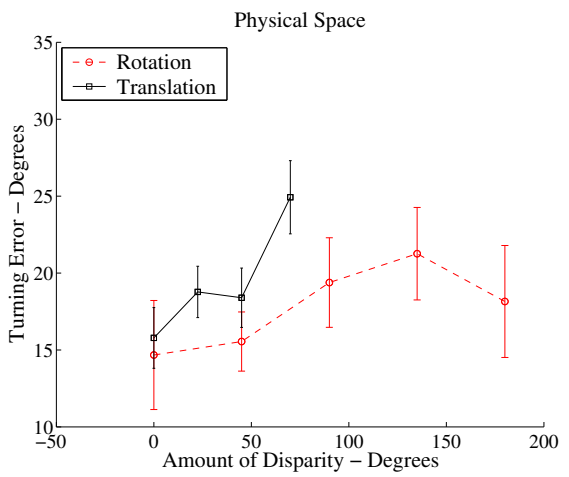

Fig. 15. Mean turning error as a function of disparity in the real world condition for Experiment 2. The red line represents the turning error in the rotational repositioning task; the black line shows the turning error in the translational repositioning task. Error bars indicate the standard error of the mean.

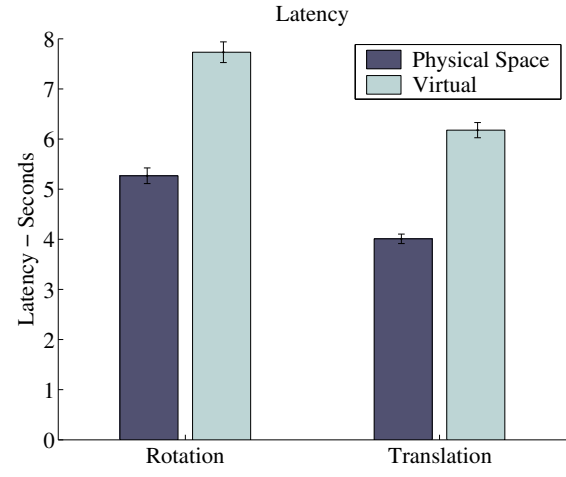

Fig. 14. Mean latency for both the translation and rotation repositioning task in the virtual and real conditions for Experiment 2. Error bars indicate the standard error of the mean.

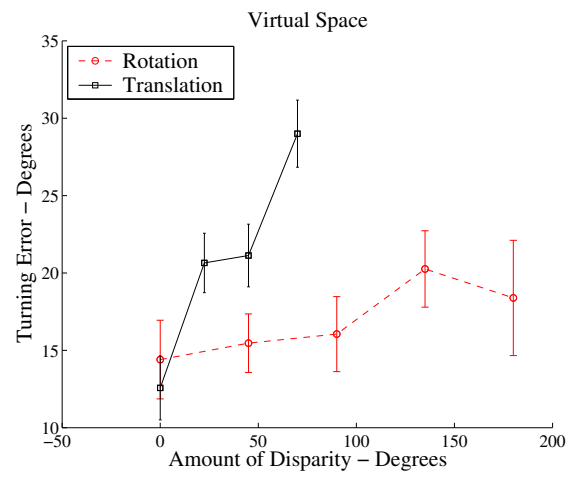

Fig. 16. Mean turning error as a function of disparity in the virtual condition for Experiment 2. The red line represents the turning error in the rotational repositioning task; the black line shows the turning error in the translational repositioning task. Error bars indicate the standard error of the mean.

To analyze these results, we again employed a model similar to that used in Experiment 1, with the substitution of a predictor of the geometry of perspective change rather than the locomotion method, and having the same number of degrees of freedom. Results were again considered significant if $p<.05$. Tables III and IV show the analysis of variance results for this experiment.

Analogous to Experiment 1, there are significant main effects of disparity and geometry on both the turning errors and latencies. Like Experiment 1, lower disparities lead to faster and more accurate performance. The main effect of geometry is, however, an ambiguous finding, since turning errors increase but latencies decrease, indicating that speed-accuracy trade-offs are being made, a point discussed earlier. For latencies, there are significant 


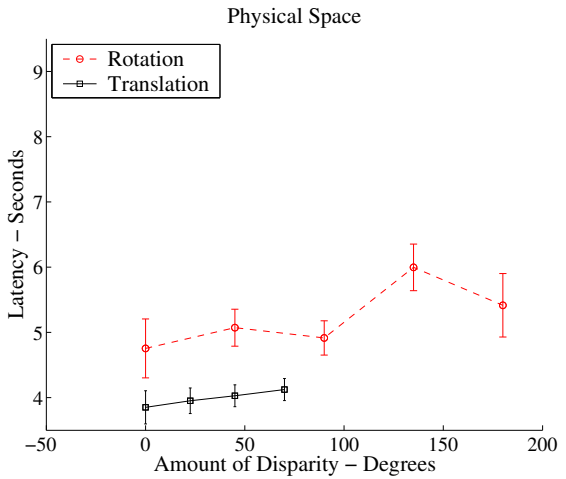

Fig. 17. Latency as a function of disparity in the real world condition for Experiment 2. The red line represents the latency in the rotational repositioning task; the black line shows the latency in the translational repositioning task. Error bars indicate the standard error of the mean.

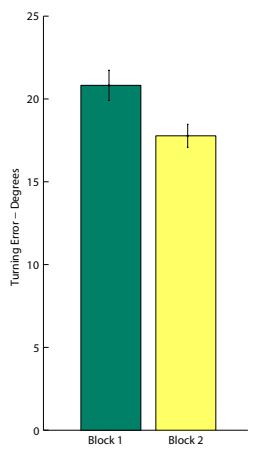

Fig. 19. This figure shows the average turning error in the first block and second block for Experiment 2. The error bars represent standard errors of the mean.

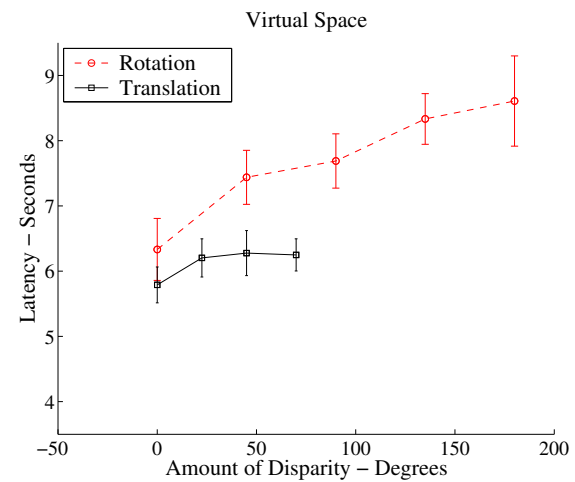

Fig. 18. Latency as a function of disparity in the virtual condition for Experiment 2. The red line represents the latency in the rotational repositioning task; the black line shows the latency in the translational repositioning task. Error bars indicate the standard error of the mean.

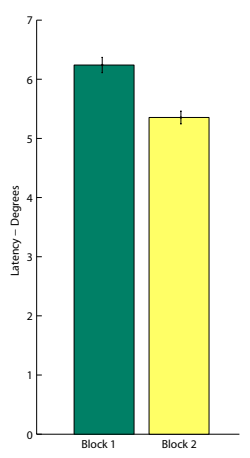

Fig. 20. This figure shows the average latency in the first block and second block for Experiment 2. The error bars represent standard errors of the mean.

main effects of the visual input mode and of learning block. As measured by latency, performance in the real world is significantly faster that performance in the virtual environment. For turning errors, there is a significant interaction between disparity and the geometry of the perspective change. This interaction indicates that disparity has a larger effect on the translation condition than the rotation condition.

\section{DISCUSSION}

This paper presents two experiments that were conducted to assess the degree to which representation-based judgments of perspective were functionally similar across conditions where the input to the representation resulted from freely viewing the physical environment versus a virtual rendering of the same environment viewed over an HMD. Judgments were assessed in terms of errors and latencies, both of which were free to vary. These 


\begin{tabular}{lrrrrr}
\hline \hline & d.f. & PartialSS & \multicolumn{1}{c}{$M S$} & \multicolumn{1}{c}{$F$} & \multicolumn{1}{c}{$p$} \\
\hline Disparity* & 7 & 18463 & 2638 & 4.6 & $<.01$ \\
Visual Input & 7 & 3610 & 516 & 0.9 & .51 \\
Geometry* & 7 & 22802 & 3257 & 5.6 & $<.01$ \\
Block & 7 & 6022 & 860 & 1.5 & .17 \\
Disparity $\times$ Visual Input & 3 & 1096 & 365 & 0.6 & .60 \\
Disparity $\times$ Geometry* & 3 & 8654 & 2885 & 5.0 & $<.01$ \\
Visual Input $\times$ Geometry & 3 & 2410 & 803 & 1.4 & .25 \\
Disparity $\times$ Block & 3 & 599 & 200 & 0.3 & .79 \\
Visual Input $\times$ Block & 3 & 1576 & 525 & 0.9 & .44 \\
Geometry $\times$ Block & 3 & 798 & 266 & 0.5 & .71 \\
Disparity $\times$ Visual Input $\times$ Geometry & 1 & 729 & 729 & 1.3 & .26 \\
Disparity $\times$ Visual Input $\times$ Block & 1 & 272 & 272 & 0.5 & .49 \\
Disparity $\times$ Geometry $\times$ Block & 1 & 221 & 221 & 0.4 & .54 \\
Visual Input $\times$ Geometry $\times$ Block & 1 & 557 & 557 & 1.0 & .33 \\
\hline
\end{tabular}

Table III. Analysis of Variance for turning error in Experiment 2. The $d$.f. column gives the number of coefficients of terms including that term in the regression model. Other columns are standard statistical measures. Significant main effects and interactions are indicated with an asterisk $(*)$.

\begin{tabular}{lrrrrc}
\hline \hline & $d . f$. & PartialSS & $M S$ & $F$ & $p$ \\
\hline Disparity* & 7 & 287 & 41 & 3.8 & $<.01$ \\
Visual Input* & 7 & 2482 & 355 & 32.8 & $<.01$ \\
Geometry* & 7 & 382 & 55 & 5.1 & $<.01$ \\
Block* & 7 & 430 & 61 & 5.7 & $<.01$ \\
Disparity $\times$ Visual Input & 3 & 41 & 14 & 1.3 & .28 \\
Disparity $\times$ Geometry & 3 & 14 & 5 & 0.4 & .74 \\
Visual Input $\times$ Geometry & 3 & 25 & 8 & 0.8 & .51 \\
Disparity $\times$ Block & 3 & 44 & 15 & 1.4 & .25 \\
Visual Input $\times$ Block & 3 & 44 & 15 & 1.4 & .25 \\
Geometry $\times$ Block & 3 & 54 & 18 & 1.7 & .17 \\
Disparity $\times$ Visual Input $\times$ Geometry & 1 & 1 & 1 & 0.1 & .71 \\
Disparity $\times$ Visual Input $\times$ Block & 1 & 17 & 17 & 1.6 & .21 \\
Disparity $\times$ Geometry $\times$ Block & 1 & 5 & 5 & 0.5 & .49 \\
Visual Input $\times$ Geometry $\times$ Block & 1 & 24 & 24 & 2.2 & .14 \\
\hline
\end{tabular}

Table IV. Analysis of Variance for latency in Experiment 2. The d.f. column gives the number of coefficients of terms including that term in the regression model. Other columns are standard statistical measures. Significant main effects and interactions are indicated with an asterisk (*).

measures are reasonable indicators of performance provided they are not traded off against one another.

By functional similarity we mean that variables influencing responses based on representations learned through experiencing a physical environment have similar influence on responses based on representations learned through experiencing a virtual environment. Functional similarity does not refer to the presence or lack of a main effect of the visual input type on the errors or latencies. An HMD has technical limitations that make it reasonable to expect performance in judging perspectives to be inferior when learned through an HMD than when experienced in a physical environment. Indeed, errors were marginally higher after learning a representation through a virtual environment in Experiment 1, and latencies were reliably longer in both Experiments 1 and 2. However, the visual input type 
had no interactions with any of the other independent variables in either experiment. Thus, the functional similarities or differences between physical and virtual environments are the similarities or differences in responses to the two input types based on measures of mode of locomotion, geometry of perspective change, and disparity.

Thus, this paper demonstrates that spatial learning is functionally similar in both real and virtual environments. Specifically, whether the spatial representation was gained through a virtual display or by sight in the physical world, physical locomotion was a strong aid in computing perspective changes. Moreover, perspective changes are harder with increasing disparity in both the physical and virtual environments. This result is well-known for physical environments, but had not been demonstrated before in virtual environments. Also, proprioceptive feedback from locomotion facilitates understanding of the spatial representation in virtual environments as well as physical ones. If this finding can be successfully exploited, it may prove useful in the design of navigation and way-finding interfaces for virtual simulations.

Some differences between our results and the results of [Rieser 1989] exist. The present study shows differences in the locomotion and imagination conditions for the no-disparity conditions, whereas [Rieser 1989] found identical performance. We believe that this difference occurs because in our experiments subjects were primed to expect a change in the to-be-imagined facing direction during the block of trials, so that it took them longer even when the instruction was to imagine facing their actual facing direction. Also, response times and errors in [Rieser 1989] were generally lower than our response times and errors. We note that in our experiments subjects were slower in both the physical environment and the virtual environment, and we attribute it to differences in experimental design: [Rieser 1989] used a swivel-mounted pointer that was manipulated by hand, whereas in our experiments subjects turned their bodies to a facing direction, and our subjects were always wearing an HMD in the testing condition. The authors have tested this experimental setup themselves and find that it is difficult to move significantly more quickly than our results indicate.

Earlier studies show that when operating in the physical environment, adults imagine simple translations in perspective more accurately and/or rapidly than simple rotations [Presson and Montello 1994; Rieser 1989]. However, in the present experiment the results are ambiguous. On the one hand, people judged the to-be-imagined translations more rapidly than the rotations, but their errors were larger. We hypothesize that the different pattern of results for errors and latencies reflect the strategies that some subjects reported. For the translation conditions, most subjects reported they were able to imagine themselves physically standing at the new observation point; we assume their judgments were rapid because they based them directly on their representation, and we assume they were inaccurate because they misjudged the distance of the needed translations. For the rotation condition, on the other hand, subjects reported they were not able to imagine actually facing in that direction. And so instead, they computed their answers by figuring the difference between their actual facing angle and the to-be-judged angle. The fact that they needed to figure is consistent with their slower latencies.

A final note about the main effects in performance between virtual environment and physical environment should be made, however. While there were no statistically significant differences in the accuracy with which people turned in the physical or virtual environments, people took significantly longer to accomplish a perspective change in the 
virtual environment than in the physical environment. This poorer performances in the virtual environment may be a quality of "immersion" related to the more limited field of view in the HMD, the poorer quality of rendering in the HMD (as opposed to vision), or the limited resolution of the HMD. Although a sense of immersion is difficult to define, gaining strong spatial representations in virtual environments is likely to be a critical component of it, and our future work is progressing in this direction.

\section{ACKNOWLEDGMENTS}

The authors would like to thank the reviewers for their insightful and constructive comments. We further thank Tim McNamara, Kaysi Holman, and Svetlana Eden for help and advice. This material is based upon work supported by the National Science Foundation under Grants IIS-0237621 and IIS-0121084, and by a Vanderbilt Discovery Grant. Any opinions, findings, and conclusions or recommendations expressed in this material are those of the authors and do not necessarily reflect the views of the sponsors.

\section{REFERENCES}

Brockmole, J. R. And WAng, R. F. 2003. Changing perspectives within and across environments. Cognition 87, B59-B67.

Chance, S. S., Gaunet, F., Beall, A. C., And Loomis, J. M. 1998. Locomotion mode affects updating of objects encountered during travel: The contribution of vestibular and proprioceptive inputs to path integration. Presence 7, 2, 168-178.

Easton, R. D. And Sholl, M. J. 1995. Object-array structure, frames of reference, and retrieval of spatial knowledge. Journal of Experimental Psychology 21, 483-500.

FARrell, M. J. AND Robertson, I. H. 1998. Mental rotation and the automatic updating of body-center spatial relationships. Journal of Experimental Psychology: Learning, Memory, and Cognition 24, 993-1005.

Harrell, JR., F. E. 2001. Regression Modeling Strategies. Springer Series in Statistics. Springer-Verlag, New York.

KAY, B. A. AND WARren, JR., W. H. 2001. Coupling of posture and gait: mode locking and parametric excitation. Biological Cybernetics 85, 2 (Aug.), 89-106.

Klatzky, R. L., Loomis, J. M., Beall, A. C., Chance, S. S., And Golledge, R. G. 1998. Spatial updating of self-position and orientation during real, imagined, and virtual locomotion. Psychological Science 9 , 4 (July), 293-298.

KNAPP, J. M. AND Loomis, J. M. 2004. Limited field of view of head-mounted displays is not the cause of distance underestimation in virtual environments. Presence: Teleoperators and Virtual Environments 13, $572-577$.

Loomis, J. M., Blascovich, J. J., AND BeALL, A. C. 1999. Immersive virtual environment technology as a basic research tool in psychology. Behavior Reseach Methods, Instruments, and Computers 31, 557-564.

Loomis, J. M., Klatzky, R. L., Golledge, R. G., And Philbeck, J. W. 1999. Human navigation by path integration. In Wayfinding: Cognitive mapping and other spatial processes, R. G. Golledge, Ed. Johns Hopkins University Press, Baltimore, MD, 125-151.

LoOMIS, J. M. AND KNAPP, J. M. 2003. Visual perception of egocentric distance in real and virtual environments. In Virtual and Adaptive Environments, L. J. Hettinger and M. W. Haas, Eds. ErlBaum, Mahwah, NJ, 21-46.

MAY, M. 1996. Thinking outside the body: An advantage for spatial updating during imagined versus physical self-rotation. Psychologische Beitrge 38, 418-434.

MAY, M. 2004. Imaginal perspective switches in remembered environments: Transformation versus interference accounts. Cognitive Psychology 48, 163-206.

May, M. And KLATZKy, R. L. 2000. Path integration while ignoring irrelevant movement. Journal of Experimental Psychology: Learning, Memory, and Cognition 26, 1 (Jan.), 169-186.

ACM Transactions on Applied Perception, Vol. V, No. N, DRAFT 2006. 
Mohler, B. J., Thompson, W. B., Creem-Regehr, S., Pick, H. L., Warren, W. H., Rieser, J. J., AND WILlEMSEN, P. 2004. Visual motion influences locomotion in a treadmilll virtual environment. In Symposium on Appliced Perception in Graphics and Visualization. Los Angeles, CA, 19-22.

Mohler, B. J., Thompson, W. B., Creem-Regehr, S., Willemsen, P., Pick, H. L., And Rieser, J. J. 2005. Calibration of locomotion due to visual motion in a treadmill-based virtual environment. ACM Transactions on Applied Perception. in press.

Myers, R. H., Montgomery, D. C., And Vining, G. G. 2002. Generalized Linear Models. Wiley Interscience, New York.

Newcombe, N. S. AND Huttenlocher, J. 2000. Making Space: The development of spatial representation and reasoning. MIT Press.

Oman, C. M., Shebilske, W. L., Richards, J. T., Tubr, T. C., Beall, A. C., and Natapoff, A. 2002. Three dimensional spatial memory and learning in real and virtual environments. Spatial Cognition and Computation 2, 355-372.

Philbeck, J. W., Klatzky, R. K., Behrmann, M., Loomis, J. M., And Goodridge, J. 2001. Active control of locomotion facilitates nonvisual navigation. Journal of Experimental Psychology: Human Perception and Performance 27, 141-153.

Plumert, J. M., Kearney, J. K., And Cremer, J. F. 2004. Child's perception of gap affordances: Bicycling across traffic-filled intersections in an immersive virtual environment. Behavior Reseach Methods, Instruments, and Computers 75, 1243-1253.

Plumert, J. M., Kearney, J. K., Cremer, J. F., And Recker, K. 2005. Distance perception in real and virtual environments. ACM Transactions on Applied Perception 2, 216-233.

Presson, C. C. AND Montello, D. R. 1994. Updating after rotational and translational body movements: coordinate structure of perspective space. Perception 23, 1447-1455.

PsotKa, J., Lewis, S. A., And King, D. 1998. Effects of field of view on judgments of self-location. Presence 7, 4 (Aug.), 352-369.

Riecke, B. E., Heyde, M. V. D., AND BÜlthoff, H. H. 2005. Visual cues can be sufficient for triggering automatic, reflexlike spatial updating. ACM Trans. Appl. Percept. 2, 3, 183-215.

RIESER, J. J. 1989. Access to knowledge of spatial structure at novel points of observation. Journal of Experimental Psychology 15, 6, 1157-1165.

Rieser, J. J., Garing, A. E., AND YounG, M. F. 1994. Imagery, action, and young children's spatial orientation: It's not being there that counts, it's what one has in mind. Child Development 65, 1262-1278.

RIESER, J. J., GUTH, D. A., AND HILL, E. W. 1986a. Sensitivity to perspective structure while walking with vision. Perception 15, 173-188.

RIESER, J. J., GUTH, D. A., AND HILL, E. W. 1986b. Sensitivity to perspective structure while walking without vision. Perception 15, 173-188.

Satterthwaite, F. E. 1941. Synthesis of variance. Psychometrika 6, 5, 319-316.

Satterthwaite, F. E. 1946. An approximate distribution of estimates of variance components. Biometrics Bulletin 2, 6, 110-114.

Thompson, W. B., Willemsen, P., Gooch, A. A., Creem-Regehr, S. H., Loomis, J. M., And Beall, A. C. 2004. Does the quality of the computer graphics matter when judging distances in visually immersive environments. Presence: Teleoperators and Virtual Environments 13, 560-571.

Waller, D., BeAll, A. C., AND Loomis, J. M. 2004. Using virtual environments to assess directional knowledge. Journal of Experimental Psychology 24, 105-116.

WANG, R. F. 2005. Beyond imagination: Perspective change problems revisted. Psicológica 26, 25-38.

Willemsen, P., Colton, M. B., Creem-Regehr, S. H., And Thompson, W. B. 2004. The effects of head-mounted display mechanics on distance judgements in virtual environments. In Proceedings of the 1st Symposium on Applied Perception in Graphics and Visualization. 36-48.

Willemsen, P. AND Gooch, A. A. 2002. Perceived egocentric distances in real, image-based and traditional virtual environments. In IEEE Virtual Reality. 79-86.

WiTMER, B. G. AND SADOWSKI, W. J. J. 1998. Nonvisually guided locomotion to a previously viewed target in real and virutal environments. Human Factors 40, 478-488.

WRAGA, M. 2003. Thinking outside the body: An advantage for spatial updating during imagined versus physical self-rotation. Journal of Experimental Psychology: Learning, Memory, and Cognition 29, 993-1005.

ACM Transactions on Applied Perception, Vol. V, No. N, DRAFT 2006. 
Wraga, M., Creem-Regehr, S. H., And Proffitt, D. R. 2004. Spatial updating of virtual displays during self- and display rotation. Memory and Cognition 32, 3 (Apr.), 399-415.

WU, B., OoI, T. L., AND HE, Z. J. 2004. Perceiving distance accurately by a directional process of integrating ground information. Nature 428, 73-77. 